\title{
Investigación cualitativa de las expectativas de médicos generales y especialistas sobre el rol del radiólogo
}

\author{
Qualitative research of general practitioners' and specialists' \\ expectations of the role of the radiologists
}

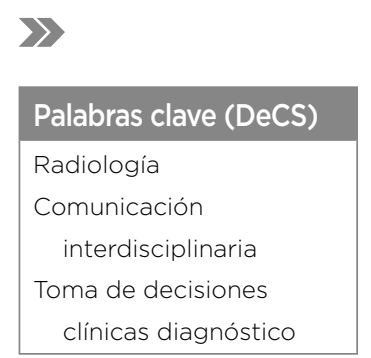

Key words (MeSH)

Radiology

Interdisciplinary

communication

Clinical decision-making diagnosis
'Médica, radióloga. Magíster en Administración de Empresas y en Telesalud. Pontificia Universidad Javeriana, Hospital Universitario San Ignacio. Bogotá, Colombia.

2Médico, radiólogo. Magíster en Epidemiología Clínica. Pontificia Universidad Javeriana, Hospital Universitario San Ignacio. Bogotá, Colombia.

3Médica, residente de Radiología. Pontificia Universidad Javeriana. Hospital Universitario San Ignacio. Especialista en Epidemiología Clínica. Universidad del Rosario. Bogotá, Colombia.

${ }^{4}$ Médica(o), radióloga(o). Pontificia Universidad Javeriana. Hospital Universitario San Ignacio. Bogotá, Colombia.

${ }^{5}$ Psicóloga, epidemióloga clínica. Pontificia Universidad Javeriana, Hospital Universitario San Ignacio. Bogotá, Colombia.

\section{Resumen}

Objetivo: Hay incertidumbre alrededor del papel actual del radiólogo en el proceso diagnóstico y terapéutico. Con el presente estudio se busca obtener información de diferentes especialistas médico-quirúrgicos sobre la opinión que tienen acerca del papel del radiólogo en la práctica médica diaria y los aspectos por mejorar de esta especialidad. Material y métodos: Se realizó un muestreo propositivo mediante una inclusión selectiva de los participantes, con base en las variables especialidad médico-quirúrgica y años de experiencia. A partir de la revisión de la literatura, se ensambló una entrevista semiestructurada y anónima para ser diligenciada. Resultados: Se realizaron y analizaron 56 entrevistas a 21 especialidades médico-quirúrgicas. Cada entrevista fue diligenciada de forma anónima. Mediante un análisis temático, se identificaron las palabras clave que se repitieron con mayor frecuencia, posteriormente se agruparon en cinco categorías (informes a la medida, integración, oportunidad, habilidades del radiólogo y participación clínica), y finalmente hicieron parte del concepto global "Trabajo en equipo". Conclusiones: La radiología es reconocida por diferentes especialistas como una especialidad fundamental en la toma de decisiones médicas, modificación de conductas terapéuticas y apoyo en abordajes quirúrgicos. En el ejercicio de la profesión se enfatizó en la importancia de un informe detallado y la capacidad de plantear diagnósticos diferenciales. Se reconoce que para proteger la especialidad, lograr un mejor desempeño y ser más eficientes se debe mejorar la participación clínica y la integración con las demás especialidades.

\section{Summary}

Objective: There is much uncertainty surrounding the current role of the radiologist in the diagnostic and therapeutic process. The purpose of this study is to obtain information directly from the different medical-surgical specialists, to know their opinion about the role of the radiologist and the aspects to be improved within this specialty. Material and methods: Participants were included based on medical-surgical speciality and years of experience. Based on the literature review, a semi-structured and anonymous interview was assembled to be completed by the participants. The custody of the data and anonymity of the information were guaranteed. Results: 56 interviews with 21 medical-surgical specialties were conducted and analyzed. Each interview was completed anonymously. According to a thematic analysis of the interviews, somthe most frequently repeated key words were identified, which in turn were grouped into five categories (tailored reports, integration, opportunity, radiologist skills and clinical participation), and finally became part of the overall concept "Teamwork". Conclusion: Radiology is recognized by various specialists as a fundamental specialty when it comes to supporting medical decision-making, modification of therapeutic approaches, and support in surgical approaches. Within the practice of the profession, the importance of a detailed report and the ability to propose differential diagnoses was emphasized. It was recognized that to protect the specialty, achieve better performance and to be more efficient, clinical participation and integration with other specialties must be improved.

\section{Introducción}

La radiología es una especialidad relativamente nueva, de evolución constante. Durante la primera mitad del siglo XX, prácticamente una sola modalidad diagnóstica constituyó su objetivo principal, los rayos $\mathrm{X}$. Estos estudios eran muy difíciles de interpretar para otros especialistas o médicos generales, ya que no tenían un adecuado conocimiento (1). Dada la necesidad que existía para la interpretación de estos estudios, entre 1920 y 1942, se consolidó como una especialidad de la medicina.

Hoy en día, existen múltiples modalidades de imágenes diagnósticas y procedimientos terapéuticos mínimamente invasivos que disminuyen los riesgos del paciente y ofrecen distintas soluciones con tecnología de punta (2). En consecuencia, el interés de otras áreas 
de la medicina en la radiología ha crecido exponencialmente, y por lo tanto la exigencia para el radiólogo, ya que este es pieza clave para el diagnóstico y para la toma de decisiones terapéuticas.

Sin embargo, hay mucha incertidumbre alrededor del papel actual del médico radiólogo en el proceso diagnóstico y terapéutico de los pacientes. Su concepto y participación es muy variable en el ámbito médico entre las diferentes especialidades. Con el presente estudio se busca obtener información directa de distintos especialistas médicoquirúrgicos, en el contexto de un hospital universitario, para conocer la opinión que tienen acerca del papel del radiólogo y los aspectos por mejorar de la especialidad.

\section{Material y métodos}

Enfoque metodológico de la investigación: El estudio se clasificó como de naturaleza descriptiva, con un enfoque cualitativo (3-5).

Tipo de estudio: Estudio cualitativo descriptivo de baja inferencia, basado en entrevistas semiestructuradas, generadas a partir de los resultados de la revisión de la literatura. La elección de este diseño se sustenta en que proporciona respuestas directas a preguntas $(6,7)$ sobre cómo se sienten las personas con respecto a situaciones particulares, qué razones tienen para sentirse de esa forma, quién y cómo se usan servicios o funciones particulares.

Variables para considerar: En la búsqueda de la literatura se encontró que hay algunos elementos que pueden ser relevantes en la forma en que las diferentes especialidades perciben la radiología y el papel del radiólogo. Esto puede generar diferentes imaginarios, expectativas, necesidades y deseos de participación y trabajo con el radiólogo o con sus productos (informe radiológico). Unas de las principales variables por considerar fueron el tipo de especialidad médico-quirúrgica y el nivel de entrenamiento del especialista (años de experiencia).

Selección de participantes: Se realizó un muestreo propositivo mediante una inclusión selectiva de los participantes, con base en las variables previamente descritas especialidad médico-quirúrgica y años de experiencia: senior más de 10, intermedio de 5 a 10 y junior menos de 5 años de experiencia. Se eligieron casos para crear un grupo rico en información específica que pudiera revelar e iluminar patrones de grupos importantes. Se realizaron 56 entrevistas semiestructuradas a especialistas médico-quirúrgicos. La estrategia de selección que se empleó fue de variación máxima, la cual seleccionó a propósito una amplia gama de casos para obtener variaciones en las dimensiones de interés con dos propósitos; el primero, documentar la diversidad y el segundo, identificar patrones comunes relevantes que son generales en las dimensiones de interés.

Tamaño de muestra y punto de parada de recolección de datos: El tamaño de muestra en el estudio cualitativo tiene la tarea de obtener una gran cantidad y calidad de información para ver y analizar en profundidad el tema de interés. Esto es posible a partir de tamaños de muestra que pueden variar dependiendo de la profundidad a la que se quiere llegar. Algunos autores dedicados a la investigación cualitativa han propuesto un número determinado de sujetos con el cual se pueda tener información suficiente para responder la pregunta. Algunos autores como Morse et al. (1998) (8) o Bernard et al. (2000) (9), han propuesto un mínimo de 30 sujetos cuando se trata de un estudio etnográfico. Por el contrario, para estudios fenomenológicos Creswell et al. (1998) (10) e inclusive, Morse et al. (5), han propuesto un mínimo de cinco a seis sujetos. De forma similar, otros, como Guest et al. (2006) (10), han sugerido que con 12 sujetos se pueden obtener resultados similares a los que se tendrían una vez alcanzado el punto de saturación. Para el estudio se contempló la posibilidad de llegar a un punto de saturación, lo cual podría ocurrir con menos de 63 sujetos preestablecidos.

Método de recolección de datos: La entrevista es una de las estrategias que más se utilizan en los estudios cualitativos. A partir de la revisión de la literatura, se ensambló una entrevista semiestructurada y anónima para ser diligenciada por los participantes. Se garantizó la custodia de estos datos y el carácter anónimo de la información. La entrevista incluyó 15 preguntas abiertas, distribuidas en 5 categorías, las cuales fueron: participación del radiólogo en el diagnóstico y tratamiento, trabajo en equipo, uso de los reportes e interpretación de las imágenes, rol del departamento de radiología y reemplazo del radiólogo (anexo).

\section{Resultados}

Se realizaron 56 entrevistas asi: 20 a especialistas junior, 18 a intermedio y 18 a senior, pertenecientes a 21 especialidades médicoquirúrgicas en el Hospital Universitario donde trabajan los autores (tabla 1). Las entrevistas fueron aplicadas por estudiantes de medicina de último año, de forma anónima.

\section{Tabla 1. Número de entrevistas según la especialidad médico-quirúrgica}

\begin{tabular}{|c|c|c|c|}
\hline $\begin{array}{l}\text { Especialidades } \\
\text { quirúrgicas }\end{array}$ & $\begin{array}{l}\text { Número de } \\
\text { especialistas }\end{array}$ & $\begin{array}{l}\text { Especialida- } \\
\text { des médicas }\end{array}$ & $\begin{array}{l}\text { Número de } \\
\text { especialistas }\end{array}$ \\
\hline $\begin{array}{c}\text { Cirugía de } \\
\text { cabeza y cuello }\end{array}$ & 2 & Nefrología & 3 \\
\hline $\begin{array}{c}\text { Cirugía } \\
\text { gastrointestinal }\end{array}$ & 3 & Neurología & 2 \\
\hline Cirugía de mama & 3 & $\begin{array}{c}\text { Otorrinolaringo- } \\
\text { logía }\end{array}$ & 3 \\
\hline Cirugía general & 3 & Pediatría & 3 \\
\hline Cirugía de tórax & 3 & Urgencias & 3 \\
\hline Cirugía vascular & 2 & $\begin{array}{c}\text { Gastro- } \\
\text { enterología }\end{array}$ & 2 \\
\hline Ginecología & 3 & Geriatría & 3 \\
\hline Neurocirugía & 2 & Medicina crítica & 2 \\
\hline Ortopedia & 3 & $\begin{array}{l}\text { Medicina } \\
\text { interna }\end{array}$ & 3 \\
\hline \multirow[t]{3}{*}{ Urología } & 3 & $\begin{array}{l}\text { Medicina } \\
\text { general }\end{array}$ & 3 \\
\hline & & Endocrinología & 2 \\
\hline & n 27 & & $n 29$ \\
\hline
\end{tabular}

Del análisis de las entrevistas se obtuvieron algunas palabras clave, por ejemplo, lectura apropiada de las imágenes, orientación tratamiento, trabajo interdisciplinario, comunicación interdisciplinaria, calidad del informe radiológico, disponibilidad, participación clínica, etc. que, a su vez, se agruparon en cinco categorías, las cuales finalmente hicieron parte de un concepto clave: "Trabajo en equipo". 
La primera categoría para abordar fue la necesidad de tener "informes a la medida", es decir, informes dirigidos al especialista según lo que "necesita" que el radiólogo le informe. Dentro de esta categoría se resaltó la necesidad de correlacionar los hallazgos imagenológicos con la historia clínica del paciente y, junto con esto, se le dio importancia a la información clínica brindada por el especialista en la solicitud del estudio, con el fin de hacer un informe más dirigido. Sobre este aspecto, el especialista intermedio de Ortopedia afirmó: “[...] sería ideal el aporte de mediciones según patología, sin embargo, creo que sería según solicitud individual de cada caso". Muchas especialidades plantearon la importancia de tener informes sistemáticos de acuerdo con la especialidad que sean precisos, es decir, que contengan clasificaciones, escalas y medidas que sean fundamentales a la hora de tomar decisiones en el manejo médico del paciente.

Entre los aspectos más importantes que debería contener el informe, según los especialistas, es la capacidad de acercar al clínico a un diagnóstico específico o a diferentes diagnósticos diferenciales. Refiriéndose a esto, el especialista intermedio de Medicina crítica dijo: "La principal utilidad del radiólogo es crear diagnósticos diferenciales alternos". Finalmente, se enfatizó que los informes a la medida van estrechamente relacionados con la experiencia del radiólogo, a lo cual el especialista senior de Urología mencionó “[...] está demostrado que la experiencia de la persona que lee el estudio es fundamental, entre más especializado sea es mejor".

La siguiente categoría es la "participación clínica", donde se espera que el radiólogo haga parte activa del manejo del paciente y de su seguimiento, que a su vez, le permitirá retroalimentarse. Se cree y espera que el radiólogo sea un apoyo fundamental en la toma de decisiones terapéuticas, orientando conductas quirúrgicas, abordaje y extensión de la cirugía y modificando tratamientos; el especialista junior de Medicina interna afirmó “[...] el radiólogo hace parte fundamental de la toma de decisiones terapéuticas".

En cuanto a la "integración”, se espera y desea que el radiólogo sea más que un simple consultor y se integre con los equipos de trabajo, mediante un mayor aporte académico interdisciplinario: asistiendo a juntas y reuniones académicas, discutiendo casos difíciles y abriendo espacios para la formación de otras especialidades a través de jornadas de actualización y rotaciones con otros residentes. Se hizo énfasis en la necesidad de tener cada vez más y mejores canales de comunicación, a ello se refirieron muchas especialidades como "ir más allá del informe". Con respecto a lo anterior, el especialista intermedio de Urología afirmó: "es más útil la opinión que el reporte como tal [...]”. Mediante una mejor integración, se busca optimizar el abordaje del paciente y fortalecer el aporte académico multidisciplinar. Nuevamente, se resaltó la importancia de un radiólogo experto que mejore la integración y los canales de comunicación; frente a este punto, el especialista intermedio de Ortopedia afirmó: "sería ideal un radiólogo experto, no solo para los pacientes, sino para la formación de los residentes y la formación continua de los especialistas".

En la categoría de "habilidades del radiólogo", se reconocieron muchas cualidades que se esperan del radiólogo, entre ellas: la experiencia en la lectura de las imágenes; el cirujano senior de Cabeza y cuello aseguró: "el radiólogo tiene el ojo experto sobre las imágenes y esto es trascendental para definir abordaje [...]". Se suma a esto la calidad humana, la capacidad de asociar los hallazgos con la historia clínica, el perfil docente teniendo en cuenta que se trata de un hospital universitario, la precisión diagnóstica, la comunicación más allá del informe y la disponibilidad. En cuanto a este último aspecto, muchas especialidades manifiestan que la principal debilidad de la radiología radica en su aislamiento.

En la categoría "habilidades del radiólogo" se encontraron dos subdivisiones: radiología intervencionista y el concepto reciente de la inteligencia artificial. Diferentes especialidades perciben la radiología intervencionista como aquella subespecialidad que más comunicación interdisciplinaria tiene con el resto de las especialidades médicoquirúrgicas y se enfatiza en el impacto positivo que esta ha tenido. A esto, el especialista intermedio de Medicina crítica opinó: “A través de la radiología intervencionista, el impacto del radiólogo ha tomado mayor importancia"; del mismo modo, se resaltó el gran aporte de esta subespecialidad en cuanto a la versatilidad de procedimientos que ofrece, lo cual, a su vez, mejora al desenlace médico de los pacientes.

El otro aspecto importante que se evaluó es el concepto que tienen los especialistas sobre la posibilidad de que la labor del radiólogo sea reemplazada por inteligencia artificial; y se encontró que la mayoría de las especialidades consideraron que la peor desventaja de esto sería la pérdida del juicio clínico. Sin embargo, se enfatizó en que se deben mejorar algunos aspectos de la especialidad para evitar su reemplazo en un futuro, dentro de las cuales se encontraron una mayor participación clínica y mejor comunicación interdisciplinaria, cualidades que una máquina no podría desarrollar. En cuanto a este tema, el especialista intermedio de Nefrología comentó: "[...] fortalecer áreas de comunicación puede hacer que el radiólogo entre a formar parte del equipo, lo que no puede hacer una máquina".

La última categoría es "la oportunidad", en la cual se mencionaron los problemas a la hora de realizar procedimientos intervencionistas y diferentes estudios y en la lectura de estos últimos. En la lectura de estudios se resaltó la importancia de la disponibilidad del radiólogo, es decir, que la especialidad pueda afrontar la gran demanda de estudios que actualmente hospitales de alta complejidad presentan. En general, se solicitó una mejor comunicación de informes críticos de forma directa y verbal; el especialista junior de Urología mencionó: "la comunicación es más eficiente cuando se habla directamente con el radiólogo que el informe".

\section{Discusión}

La percepción de los médicos de otras especialidades médicoquirúrgicas en varios aspectos del trabajo del radiólogo en nuestro hospital es similar a lo identificado en la literatura.

En "informes a la medida" se menciona la importancia de la información clínica para la interpretación de las imágenes diagnósticas por medio de sistemas de información $(2,8-12)$ o con otras estrategias, como en el estudio de Gunderman et al. (9), donde se describe que el aporte de información clínica reduce significativamente los tiempos de diagnóstico y mejora la indicación de estudios apropiados. Boonn et al. (11) encontraron que los radiólogos quieren más información clínica; sin embargo, no buscan la información por el tiempo que se debe invertir para esto. Barron et al. (13) concluyeron que una intervención simple, como educar al personal que solicita los estudios, mejoró la calidad de la información clínica y, a su vez, los tiempos de respuesta. También, otros autores, como Hanna et al. (14), Schreiber et al. (15) y Doubilet 
et al. (16), plantearon la problemática que representa la pobre o nula información clínica en la interpretación de los estudios imagenológicos, en cuanto al aumento de los falsos positivos y en los tiempos de espera.

Otros autores, como Schwartz et al. (17), han afirmado que los informes estructurados tienen un mejor contenido y una mayor claridad que los informes convencionales, al igual que la percepción de los especialistas de la institución, quienes resaltan la importancia de informes más específicos según sus necesidades. También, autores como Khorasani et al. (18) y Rosenkrantz et al. (19) han encontrado que la terminología comprensible en los informes es muy importante, ya que beneficia las conductas terapéuticas. Por otro lado, se ha descrito que mencionar los hallazgos incidentales menores puede generar ansiedad en el paciente y la solicitud de estudios no necesarios; los entrevistados de este trabajo mencionaron que al estructurar los informes el lenguaje sería claro para todas las partes. Herramientas como glosarios realizados y conocidos por diferentes especialidades podría facilitar la comunicación, como lo que ocurre con el glosario Fleisschner, el cual permite un lenguaje uniforme entre neumólogos, cirujanos de tórax y radiólogos.

En este estudio se destacó la importancia de los diagnósticos alternos dados por el radiólogo en su informe y el valor que tiene la lectura de los estudios por parte de un radiólogo especializado, aspectos que favorecen la labor del médico no radiólogo en su ejercicio profesional. Por otro lado, en cuanto a la lectura del informe por parte del paciente, un especialista en ortopedia mencionó que pueden surgir situaciones complicadas al tratar de explicarle al paciente los hallazgos descritos en un informe radiológico, la clínica que presenta y las conductas adecuadas para su patología, dadas las frases que se mencionan en el informe y el valor que el paciente les da.

En cuanto a "participación clínica" e "integración”, Ramírez et al. (20) consideran que la comunicación adecuada entre los médicos tratantes y los radiólogos es esencial en la práctica clínica de alta calidad, facilita el intercambio de opiniones y permite obtener información clínica necesaria. Wallis et al. (21) animan al radiólogo a esforzarse por mejorar la comunicación con otros especialistas. Por su parte, Bosmans et al. (22) consideran que los médicos no radiólogos esperan que el radiólogo ofrezca respuestas clínicas por medio del informe, pero que también sea posible una comunicación directa. Similiar a lo descrito por estos estudios, en nuestro estudio las diferentes especialidades quieren que el radiólogo sea parte del grupo terapéutico, que participe en las juntas de decisiones médicas de forma más activa, que siempre esté dispuesto a responder dudas y al diálogo con el resto del personal médico; esta participación interdisciplinaria y activa se destacó mucho más que la elaboración de un informe escrito.

Glazer et al. (23) se cuestionan acerca de cómo los radiologos se vuelven invisibles para los pacientes, qué factores impulsan esto y qué efecto puede tener a largo plazo. De igual forma, Kemp et al. (24) encontraron que la mayoría de radiólogos piensan que es importante la comunicación directa entre los pacientes y ellos; no obstante, refieren que se ven limitados para este fin por el tiempo y la carga de trabajo. Sin embargo, cuando se dan las oportunidades, es apropiado que el radiólogo se presente con el paciente, le explique los hallazgos de las imágenes con un lenguaje sencillo y les mencione la importancia de realizar las siguientes conductas con el especialista que corresponda.

En las "habilidades del radiólogo", se considera que su ejercicio profesional es determinante para la toma de decisiones clínicas y terapéuticas. Bosmans et al. (22) encontraron que el 85,5\% de los especialistas se sienten satisfechos con los informes y para la gran mayoría el informe radiológico detallado fue de gran ayuda en el manejo médico. Al igual que en este estudio, se destacó la idoneidad de los radiólogos, resaltando los momentos en los cuales mediante la discusión se orientaron decisiones clínicas relevantes. Así mismo, se le dio un gran valor al trabajo que desempeña el radiólogo intervencionista, el cual por medio de las técnicas mínimamente invasivas guiadas por imágenes y en tiempos rápidos ofrece opciones terapéuticas a diferentes patologías que incluso antes no tenían solución (2). Esta fortaleza es reconocida y destacada por los médicos de nuestro hospital, resaltando que existen mejores canales de comunicación con esta subespecialidad.

Por otra parte, surge también un desafío en cuanto a lo que representa la inteligencia artificial (IA) y el papel del radiólogo $(25,26)$. La IA es una herramienta que deber ser incorporada a la práctica clínica del radiólogo como un medio para mejorar la extracción de información útil, sin que esto signifique un "reemplazo". En cuanto a este aspecto, que también se indagó en el estudio aquí descrito, se observó que la percepción de los especialistas fue que la comunicación con el radiólogo y su interacción no se puede dejar en manos de aparatos.

En cuanto a "oportunidad", autores como Vivas et al. (8) destacaron como errores las fallas en la interpretación del radiólogo y otras relacionadas en el sistema, en el que destacaron la excesiva cantidad de trabajo. Es el caso de países en desarrollo, que tienen problemas con los tiempos de espera desde la adquisición de las imágenes, hasta su interpretación; en este trabajo se identificaron estos mismos inconvenientes y la necesidad de contar con vías de comunicación expeditas; al respecto, los médicos no radiólogos les dieron valor a los informes críticos comentados verbalmente de forma oportuna.

\section{Conclusiones}

La radiología es reconocida por los diferentes especialistas médicoquirúrgicos como una especialidad fundamental a la hora de tomar decisiones médicas, para la modificación de conductas terapéuticas o para apoyar abordajes quirúrgicos. En el ejercicio de la profesión se enfatiza en la importancia de un informe detallado y la capacidad de plantear diagnósticos diferenciales. Sin embargo, se reconoce que, para proteger la especialidad, lograr un mejor desempeño y ser más eficientes, se debe mejorar la participación clínica y la integración con las demás especialidades, para lo que es fundamental tener radiólogos subespecialistas, que sean expertos en cada área de la medicina.

\section{Referencias}

1. Feldman A. A sketch of the technical history of radiology from 1896 to 1920 . RadioGraphics. 1989;9(6):111328. https://doi.org/10.1148/radiographics.9.6.2685937

2. Pirnejad H, Niazkhani Z, Bal R. Clinical communication in diagnostic imaging studies: mixed-method study of pre- and post-implementation of a hospital information system. Appl Clin Inform. 2013; 4(4):541-55. https://doi.org/10.4338/ACI-2013-06-RA-0042

3. Colorafi KJ, Evans B. Qualitative descriptive methods in health science research. HERD Heal Environ Res Des J. 2016;9(4):16-25. https://doi.org/10.1177/1937586715614171

4. Bernard HR, Harvey R. Social research methods : qualitative and quantitative approaches. Sage Publications; 2000.

5. Denzin NK, Lincoln YS. Handbook of qualitative research. Thousand Oaks: Sage Publications; 1994.

6. Guest G, Bunce A, Johnson L. How many interviews are enough? Field methods 2006; 18(1):59-82. https://doi.org/10.1177/1525822X05279903

7. Doody O, Noonan M. Preparing and conducting interviews to collect data. Nurse Res. 2013;20(5):28-32. https://doi.org/10.7748/nr2013.05.20.5.28.e327. 
8. Vivas I. Errores comunes en la interpretación oncológica. Radiologia. 2018; 60:53-63. https://doi.org/10.1016/j.rx.2018.01.002

9. Gunderman RB, Phillips MD, Cohen MD. Improving clinical histories on radiology requisitions. Acad Radiol. 2001 ;8(4):299-303. https://doi.org/10.1016/S10766332(03)80498-1.

10. Renfrew DL, Franken EA, Berbaum KS, Weigelt FH, Abu-Yousef MM. Error in radiology: classification and lessons in 182 cases presented at a problem case conference. Radiology. 1992 ;183(1):145-50. https://doi.org/10.1148/radiology.183.1.1549661.

11. Boonn WW, Langlotz CP. Radiologist use of and perceived need for patient data access. J Digit Imaging. 2009 ;22(4):357-62. https://doi.org/10.1007/s10278-008-9115-2.

12. Troude P, Dozol A, Soyer P, Girard D, Martínez F, Montagne B, et al. Improvement of radiology requisition. Diagn Interv Imaging. 2014;95(1):69-75. https://doi. org/10.1016/j.diii.2013.07.002

13. Barron D, Spiegel T, Katzman GL, Haas K, Ali S. Improving clinical information on head CT requisitions from the emergency department to aid interpretation and billing efficiency. Am J Roentgenol. 2018 ;210(1):W18-21. https://doi.org/10.2214/ AJR.17.18558

14. Hanna TN, Rohatgi S, Shekhani HN, Dave IA, Johnson J-O. Clinical information available during emergency department imaging order entry and radiologist interpretation. Emerg Radiol. 2017 ;24(4):361-7. https://doi.org/10.1007/s10140-017-1488-4

15. Schreiber MH. The clinical history as a factor in roentgenogram interpretation. JAMA. 1963;185(5):399. https://doi.org/10.1001/jama.1963.03060050077027.

16. Doubilet P, Herman PG. Interpretation of radiographs: effect of clinical history. AJR. 1981;137(5):1055-8. https://doi.org/10.2214/ajr.137.5.1055.

17. Schwartz LH, Panicek DM, Berk AR, Li Y, Hricak H. Improving communication of diagnostic radiology findings through structured reporting. Radiology. 2011;260(1):174-81. http://pubs.rsna.org/doi/10.1148/radiol.11101913

18. Khorasani R, Bates DW, Teeger S, Rothschild JM, Adams DF, Seltzer SE. Is terminology used effectively to convey diagnostic certainty in radiology reports? Acad Radiol. 2003;10(6):685-8. doi: 10.1016/s1076-6332(03)80089-2

19. Rosenkrantz AB. Differences in Perceptions Among Radiologists, Referring Physicians, and Patients Regarding Language for Incidental Findings Reporting. Am J Roentgenol. 2017 ;208(1):140-3. https://doi.org/10.2214/AJR.16.16633

20. Ramírez J, Rodríguez C, Quiroz Ó, Motta G. La comunicación del radiólogo con médicos tratantes y pacientes. Acta Médica Grupo Ángeles. 2007;5(4):228-32.

21. Wallis A, McCoubrie P. The radiology report - Are we getting the message across? Clin Radiol. 2011;66(11):1015-22. https://doi.org/10.1016/j.crad.2011.05.013.

22. Bosmans J, Schrans D, Avonts D, Maeseneer J De. Communication between general practitioners and radiologists: opinions, experience, promises, pitfalls. J Belgian Soc Radiol. 2014;97(6):325. http://doi.org/10.5334/jbr-btr.127

23. Glazer GM, Ruiz-Wibbelsmann JA. The invisible radiologist. Radiology. 2011;258(1):18-22. https://doi.org/10.1148/radiol.10101447.

24. Kemp JL, Mahoney MC, Mathews VP, Wintermark M, Yee J, Brown SD. Patientcentered radiology: Where are we, where do we want to be, and how do we get there? Radiology. 2017;285(2):601-8. https://doi.org/10.1148/radiol.2017162056

25. Hosny A, Parmar C, Quackenbush J, Schwartz LH, Aerts HJWL, Edu HH. Artificia intelligence in radiology HHS Public Access. Nat Rev Cancer. 2018;18(8):500-10. https://doi.org/10.1038/s41568-018-0016-5

26. Thrall JH, Li X, Li Q, Cruz C, Do S, Dreyer K, et al. Artificial intelligence and machine learning in radiology: Opportunities, challenges, pitfalls, and criteria for success. J Am Coll Radiol. 2018;15(3):504-8. https://doi.org/10.1016/j.jacr.2017.12.026

\section{Correspondencia}

Laura Cristina Morales Cifuentes

Carrera 67 \# 106-60

Bogotá, Colombia

lauracristina25@hotmail.com

Recibido para evaluación: 4 de febrero de 2021

Aceptado para publicación: 26 de mayo de 2021 